ACCEPTED MANUSCRIPT

\title{
Rhombohedral R3c to orthorhombic Pnma phase transition induced by Y-doping in $\mathrm{BiFeO}_{3}$
}

To cite this article before publication: Monica Elisabet Graf et al 2018 J. Phys.: Condens. Matter in press https://doi.org/10.1088/1361$\underline{648 X / a a c 74 f}$

\begin{abstract}
Manuscript version: Accepted Manuscript
Accepted Manuscript is "the version of the article accepted for publication including all changes made as a result of the peer review process, and which may also include the addition to the article by IOP Publishing of a header, an article ID, a cover sheet and/or an 'Accepted Manuscript' watermark, but excluding any other editing, typesetting or other changes made by IOP Publishing and/or its licensors"
\end{abstract}

This Accepted Manuscript is @ 2018 IOP Publishing Ltd

During the embargo period (the 12 month period from the publication of the Version of Record of this article), the Accepted Manuscript is fully protected by copyright and cannot be reused or reposted elsewhere.

As the Version of Record of this article is going to be / has been published on a subscription basis, this Accepted Manuscript is available for reuse under a CC BY-NC-ND 3.0 licence after the 12 month embargo period.

After the embargo period, everyone is permitted to use copy and redistribute this article for non-commercial purposes only, provided that they adhere to all the terms of the licence https://creativecommons.org/licences/by-nc-nd/3.0

Although reasonable endeavours have been taken to obtain all necessary permissions from third parties to include their copyrighted content within this article, their full citation and copyright line may not be present in this Accepted Manuscript version. Before using any content from this article, please refer to the Version of Record on IOPscience once published for full citation and copyright details, as permissions will likely be required. All third party content is fully copyright protected, unless specifically stated otherwise in the figure caption in the Version of Record.

View the article online for updates and enhancements. 


\title{
Rhombohedral $R 3 c$ to orthorhombic Pnma phase transition induced by $\mathrm{Y}$-doping in $\mathrm{BiFeO}_{3}$
}

\author{
M E Graf ${ }^{1}$, S Di Napoli ${ }^{2,3}$, M A Barral ${ }^{2,3}$, L M Saleh Medina ${ }^{4}$, R M \\ Negri $^{4}$, M Sepliarsky $^{1}$ and A M Llois ${ }^{2,3}$ \\ ${ }^{1}$ Instituto de Física Rosario (CONICET-UNR), Rosario, Argentina \\ 2 Instituto de Nanociencia y Nanotecnología (INN CNEA-CONICET), Buenos Aires, \\ Argentina \\ ${ }^{3}$ Departamento de Física de la Materia Condensada, GlyA-CNEA, Avenida General \\ Paz 1499, (1650) San Martín, Pcia. de Buenos Aires, Argentina \\ ${ }^{4}$ Instituto de Química Física de Materiales, Ambiente y Energía (INQUIMAE), \\ Departamento de Química Inorgánica, Analítica y Química Física, Facultad de \\ Ciencias Exactas y Naturales, Universidad de Buenos Aires, Argentina
}

Keywords: $\mathrm{BiFeO}_{3}, \mathrm{Y}$ doping, phase transition, ab initio calculations

Abstract.

In this work we study, by means of $a b$ initio calculations, the structural, electronic and magnetic properties of $\mathrm{Y}$-doped $\mathrm{BiFeO}_{3}$ compounds. We determine that there is a morphotropic phase boundary at an yttrium concentration of $(18 \pm 2) \%$, where the structure changes from $R 3 c$ to Pnma. This structural transition is driven by the chemical pressure induced by the dopant.

By analyzing the evolution of the oxygen octahedral tilts we find an enhanced antiferrodistortive distortion when increasing the Y-doping, together with a reduction of the ferroelectric distorsion, that gives rise to a smaller value of the electric polarization. These cooperative effects should lead to a larger canting of the $\mathrm{Fe}$ magnetic moments and to a larger ferromagnetic response in the $R 3 c$ phase, as it is observed in the experiments.

\section{Submitted to: J. Phys.: Condens. Matter}




\section{Introduction}

Multiferroic materials, which exhibit two or more ferroic orders, have an important potential for technological applications in novel devices and sensors. Among them, the paradigmatic $\mathrm{BiFeO}_{3}$ shows multiferroicity at room temperature. It is ferroelectric below $T_{C}=1100 \mathrm{~K} \mathrm{[1],} \mathrm{with}$ spontaneaous polarization of around $90-100 \mu \mathrm{C} \mathrm{cm}^{-2}$ along the [111] pseudocubic direction [2; 3] and antiferromagnetic below $T_{N}=640 \mathrm{~K}$ [4]. It has a canted G-type antiferromagnetic ordering with a cycloid spin modulation of $620 \AA$ wavelength around the polar direction, its macroscopic magnetization averaging to zero. Besides, weak ferromagnetism has been reported in thin films and in nanoparticles of $\mathrm{BiFeO}_{3}[5 ; 6]$ and also in doped bulk compounds where $\mathrm{Bi}^{3+}$ and/or $\mathrm{Fe}^{3+}$ are substituted by other ions with comparable radii [7].

The improvement of the multiferroic properties at room temperature, the reduction of the leakage currents and the enhancement of the piezoelectric response as well as the presence of ferromagnetic order is desirable. Many works have been devoted to these goals and different strategies have been proposed. As mentioned above, the effect of doping has received considerable attention. For instance, the compressive lattice distortion induced by Co substitution at Fe sites, significantly enhances the magnetic properties attaining a saturated magnetization of $0.09 \mu_{B}$ (f.u.) at $300 \mathrm{~K}$ in $\mathrm{BiFe}_{0.95} \mathrm{Co}_{0.05} \mathrm{O}_{3}$ bulk ceramics [8]. In addition, substitution of bismuth by ions with different ionic radius, rare-earths for example, is thought to be an effective way of achieving ferromagnetism at room temperature. Moreover, since the ferroelectricity of $\mathrm{BiFeO}_{3}$ arises from the $\mathrm{Bi} 6$ s lone-pair electrons [2], chemical substitution of the perovskite's Asite can affect also the ferroelectric properties. In this sense, doping with $\mathrm{Y}^{3+}$ is expected to induce lattice distortions and to modify the magnetic and ferroelectric properties, due to the fact that $\mathrm{Y}^{3+}$ lacks a lonepair and has an ionic radius of $1.02 \AA$, while the one of $\mathrm{Bi}^{3+}$ is of $1.17 \AA$. In fact, it is experimentally observed that doping $\mathrm{BiFeO}_{3}$ nanoparticles with yttrium enhances the ferromagnetic response, also lowers the leakage currents, and supresses the formation of secondary phases usually found in $\mathrm{BiFeO}_{3}$ [9-12].

The structural variability of $\mathrm{Bi}_{1-x} \mathrm{Y}_{x} \mathrm{FeO}_{3}$ reported in the literature is remarkable. For instance, in
Ref. 9, Saleh-Medina et al. found that there is a compositional driven structural modification attributed to a rhombohedral to tetragonal phase transition for $0.1<x<0.2$ in $\mathrm{Bi}_{1-x} \mathrm{Y}_{x} \mathrm{FeO}_{3}$ nanoparticles. This is in agreement with Mishra et al., who worked with powder samples in the composition range $0<x<$ 0.15 [13]. On the other hand, Sheng et al. reported a structural transition from rhombohedral $R 3 c$ to orthorhombic Pna2 ${ }_{1}$ when increasing $x$ above 0.10 in $\mathrm{Bi}_{1-x} \mathrm{Y}_{x} \mathrm{FeO}_{3}$ polycrystalline films [14], this transition was also found by Luo et al. in ceramic samples [15]. Moreover, in Ref. 16 a structural symmetry breaking from rhombohedral $R 3 c$ to orthorhombic Pnma, at $x \simeq 0.10$, was identified in powder samples across a ferroelectric-paraelectric phase transition. In fact, it is clear that the nature and sequence of the structural phase transitions as a function of $Y$ doping in this alloy, is still an open question.

Up to the best of our knowledge there are no reported $a b$ initio calculations for the Y-doped compounds till now. In order to shed some light on the unresolved issues and taking into account the well known high predictive ability of Density Functional Theory (DFT), we study in this work the effect of Y-doping on the structural and electronic properties of bulk $\mathrm{BiFeO}_{3}$ making use of DFT calculations and comparing with experimental results, in particular those of Ref. 9.

\section{Computational Details}

We perform first-principles calculations within the framework of Density Functional Theory and the projector augmented wave (PAW) method [17], as implemented in the Vienna $a b$ initio package (VASP) [18; 19]. We explicitly treat 15 valence electrons for Bi $\left(5 d^{10} 6 s^{2} 6 p^{3}\right), 16$ for Fe $\left(3 s^{2} 3 p^{6} 3 d^{6} 4 s^{2}\right)$, 6 for $\mathrm{O}\left(2 \mathrm{~s}^{2} 2 \mathrm{p}^{4}\right)$ and 11 for $\mathrm{Y}\left(4 \mathrm{~s}^{2} 4 \mathrm{p}^{6} 4 \mathrm{~d}^{1} 5 \mathrm{~s}^{2}\right)$. The generalized gradient approximation (GGA) in the parametrization of Perdew, Burke and Ernzerhof (PBE) [20] is used. We include a Hubbard term with $\mathrm{U}=4 \mathrm{eV}$ for a better treatment of the Fe 3d-electrons in $\mathrm{BiFeO}_{3}$ [3]. All the DFT calculations are performed using a $500 \mathrm{eV}$ energy cutoff in the plane waves basis.

We work with a $2 \times 2 \times 2$ supercell containing 8 unit cells of the pseudo-cubic perovskite, as sketched in the left panel of figure 1. This supercell is compatible with the structural distortions 
that characterize the low-symmetry phases of many perovskites, allowing us to study the stability of different structural phases that might be present when doping $\mathrm{BiFeO}_{3}$ with yttrium (i.e.: R3c, Pnma, Pna $2_{1}$ and $P 4 \mathrm{~mm}$ symmetries). With respect to the magnetic structure in this material, the noncollinearity of the Fe magnetic moments is known to be quite minimal and, therefore we approximate the magnetic configuration with a collinear model, when exploring the structural phase transitions. The most relevant antiferromagnetic (AFM) orders shown by the Fe atoms in the considered oxides, G-AFM (AFM coupling between first nearest neighbours), A-AFM (ferromagnetic (FM) coupling within the (001) planes and AFM between adjacent planes) and C-AFM (AFM within the (001) planes and FM between adjacent planes), can also be described with the used supercell. With this 40-atom unit cell, we are able to take into account the following $\mathrm{Y}$ concentrations: $12.5 \%, 25 \%$, $37.5 \%$ and $50 \%$.

In order to explore lower Y-concentrations, as those achieved in the experiments [9], we also perform calculations in a $1 \times 1 \times 2$ hexagonal $R 3 c$ cell containing 12 formula units, as it is shown in the right panel of figure 1 . Within this 60 -atom unit cell, we are able to take into account the following values of Y-doping: $8.33 \%, 16.66 \%, 25 \%, 33 \%$ and $41.67 \%$ and $50 \%$.

It is worthy to mention that the insertion of $Y$ dopants in $\mathrm{BiFeO}_{3}$, within all the studied symmetries, produces small distortions in the corresponding structures, as we do not introduce any constraints, neither on the angles nor in the bonding-lengths, when performing the calculations. Nevertheless, we keep the corresponding symmetry labels (i.e.: $R 3 c$, Pnma, Pna2 1 and P4mm) in order to trace back the original structural prototypes, as the induced distortions are slight.

For the 40-atom unit cell we use a $5 \times 5 \times 5$ Monkhorst-Pack $k$-point grid centered at the $\Gamma$-point to evaluate integrals within the Brilloin zone (BZ), while a $5 \times 5 \times 2$ grid is used for the hexagonal cell. The structural relaxations are performed until the forces on each ion are less than $0.01 \mathrm{eV} \AA$.

\section{Results and discussion}

As a first step, we study the relative stability of the R3c, Pnma, Pna2 1 and P $4 m m$ phases of bulk $\mathrm{BiFeO}_{3}$ as a function of volume and magnetic configuration by considering the mentioned magnetic structures (FM, A-AFM, G-AFM and C-AFM) reported in the literature [21]. In figure 2 the total energy of the different structures is plotted as a function of unit cell volume. The data are fitted by the Birch-Murnaghan's
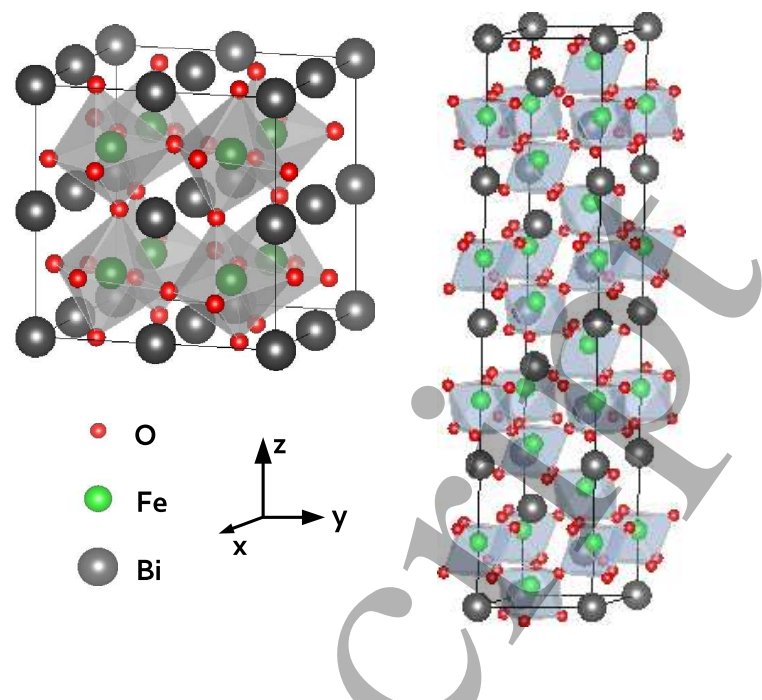

Figure 1. (Color online) Schematic representation of the considered unit cells for $\mathrm{BiFeO}_{3}$ : 40-atom unit cell (left) and 60-atom unit cell (right).

equation of states. It can be seen from figure 2 (a) that the ground state of pristine $\mathrm{BiFeO}_{3}$ corresponds to an R3c G-AFM phase, in agreement with the literature $[22 ; 23]$. The optimized lattice parameters for the ground state agree well with the experimental values and with previous DFT calculations, as it can be seen from table 1 .

We find a compression-induced structural transition from rhombohedral $R 3 c$ to orthorhombic Pnma at an estimated pressure of $P=4.8 \mathrm{GPa}$, in agreement with previous DFT calculations [3; 24]. On the other hand, the system presents another transition under negative pressure. The volume expansion induces a transformation from $R 3 c$ to $P n a 2_{1}$, together with a magnetic transition from the G-AFM to the CAFM configuration. The last phase displays a tetragonal type of structure with a remarkable elongation of the out-of-plane cell parameter, and thus an enhanced $c / a$ ratio $(c / a=1.27)$. This (negative) pressureinduced transformation is consistent with the socalled supertetragonal phase observed in $\mathrm{BiFeO}_{3}$ films grown on compressive substrates $[3 ; 25 ; 26]$.

In the recent experimental work of Ref. 9 the effects of $\mathrm{Y}$ doping on the structural, magnetic and electric properties of $\mathrm{BiFeO}_{3}$ nanoparticles have been studied [9]. The values of $\mathrm{Y}$ doping considered were $x=0 ., 0.05,0.10$ and 0.20 . In that work it was determined, by X-ray diffraction (XRD), that all the samples were essentially in a crystalline single phase with perovskite structure. For $x \leq 0.10$ the compound was found to keep the $R 3 c$ symmetry while for $x=$ 0.20 a different phase was observed. The distortion of the XRD pattern with increasing $Y$ substitution was in agreement with the presence of a structural 
phase transition, similar to the ones reported in many previous works when doping with rare earths [24; 2729]. In addition to this phase transition, Saleh-Medina et al. found a change in the magnetic behavior at room temperature from weak-ferromagnetism ( $w F M)$ for $x \leq 0.10$ to superparamagnetic-like for $x=0.20$.
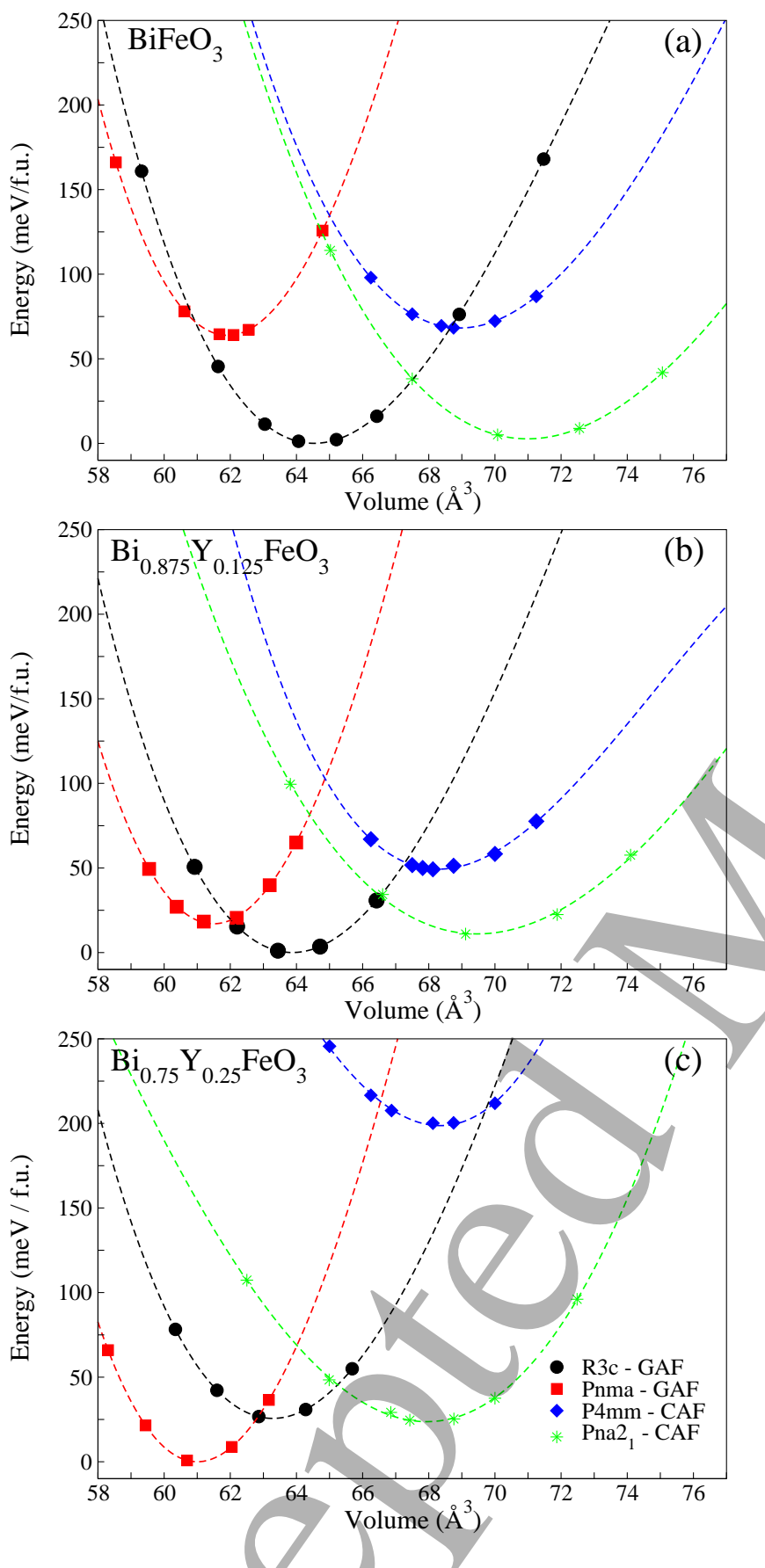

Figure 2. (Color online) Energy difference versus volume curves for the most stable phases of $\mathrm{Bi}_{1-x} \mathrm{Y}_{x} \mathrm{FeO}_{3}$. The zero energy is the corresponding ground state for each $x$-value. (a) corresponds to $x=0$, (b) to $x=0.125$ and (c) to $x=0.25$. The continous lines correspond to the fitted Birch-Murnaghan's equation of states for each case.
In order to get insight into the observed phase transition, we study the structural and electronic properties of the ground state for different $Y$ concentrations considering the same crystal structures and magnetic configurations as for pristine $\mathrm{BiFeO}_{3}$. In figures 2 (b) and (c) the total energy curves as a function of unit cell volume are presented, for $x=0.125$ and 0.25 , respectively. For simplicity, we only show the curves that correspond to the more stable magnetic configurations for each structural symmetry. It is worth mentioning that for $x \geq 0.25$, there are many possible arrangements for the dopant atoms. For each dopant-concentration within all the studied structural phases we analyse the incidence of several dopantdistributions in the fully relaxed total energy. We find that the different patterns give rise to an error lower than $6 \mathrm{meV} / \mathrm{f} . \mathrm{u}$. in the corresponding energy minimum. In Fig. 2 c) we show the calculated energies for the more stable pattern in each phase.

While the ground state for $x=0.125$ is still $R 3 c$ (See figure 2 (b)), it is clear from figure 2 (c) that the most stable phase corresponding to $x=$ 0.25 is the Pnma with G-AFM magnetic ordering. This result is robust against a dopant-configuration change. Therefore, the calculations show that a structural phase transition takes place between $x=$ 0.125 and $x=0.25$ and that it clearly corresponds to a rhombohedral (R3c) to an orthorhombic (Pnma) transformation.

In figures 3(a) and (b) we show the experimental XRD patterns reported by Saleh Medina et al. [9] for the different Y-concentrations and the $a b$ initio simulated patterns corresponding to the different structural symmetries, respectively. As it can be observed, for the pristine $\mathrm{BiFeO}_{3}$, the calculated main peaks of the $R 3 c$ structure are located at angles $2 \theta$, that agree well with the corresponding experimental ones (See also figure 3(c)). When comparing the XRD patterns for $x=0,0.05$ and 0.10 in figure 3(a) a shift of the main peaks towards higher values of $2 \theta$ is observed, consistent with a shrinking of the unit cell, which also is obtained in the calculations (see table 2). The main features observed in the pattern corresponding to $x=0.20$ that could be correlated to a Pnma symmetry are: (i) the development of an incipient peak around $2 \theta=25^{\circ}$, not present at lower concentrations; (ii) the broadening of the peak located around $2 \theta=32^{\circ}$ compatible with splittings seen in the Pnma case, indicating a lowering of the symmetry and (iii) the localization of the main peaks experimentally obtained matches well the simulated ones (See figure 3(d)). Therefore, the $a b$ initio results are consistent with the experimentally observed phase transition at $0.10<x \leq 0.20$, confirming that the induced phase is the Pnma. 

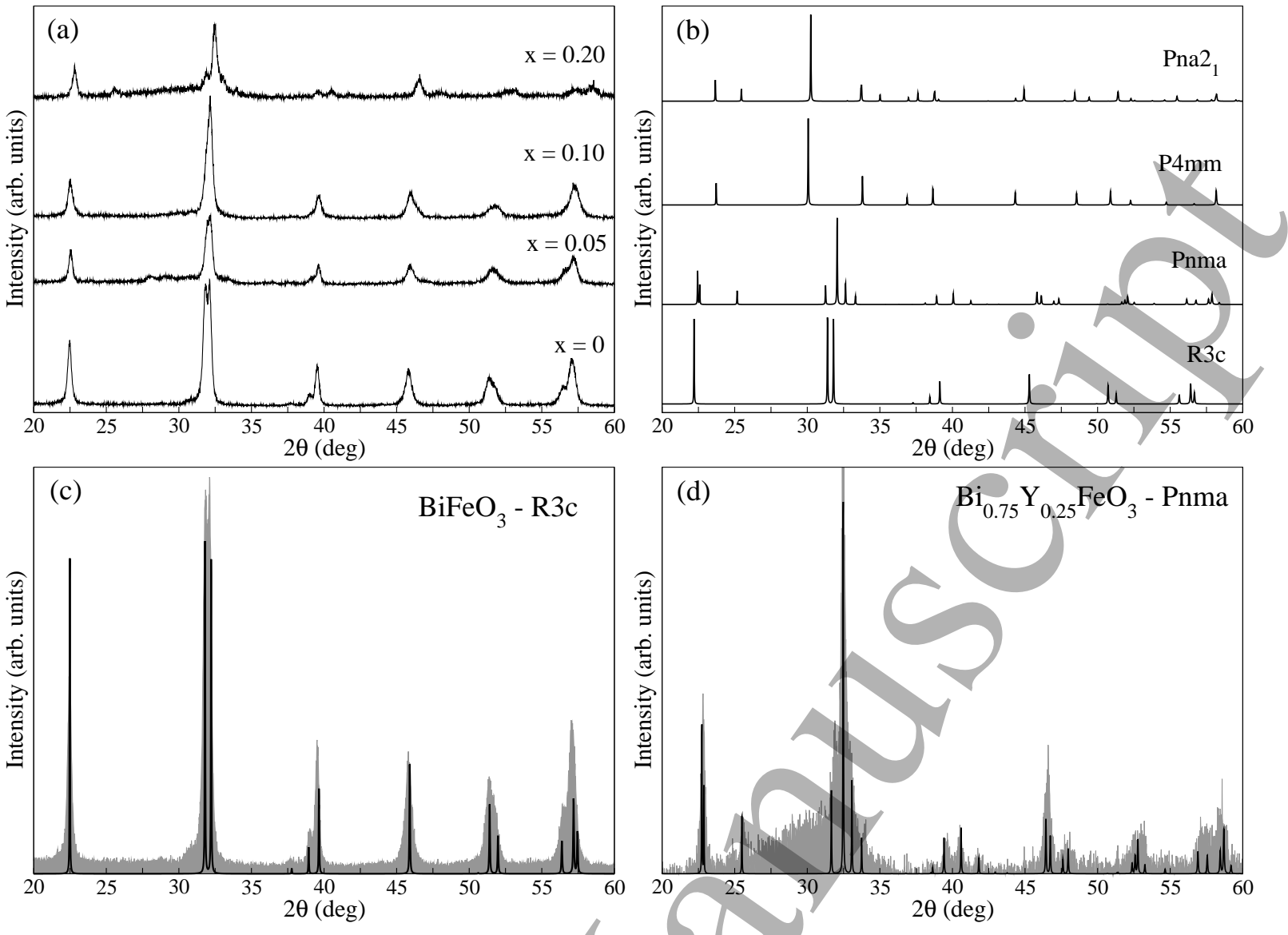

Figure 3. XRD patterns corresponding to (a) Ref. 9, experimentally obtained for different Y-concentrations, (b) ab initio simulated for the different symmetries, (c) (bold) ground state of $\mathrm{BiFeO}_{3}$ calculated in this work and (shadow) pristine $\mathrm{BiFeO}_{3}$ obtained in Ref. 9, (d) (bold) ground state of $\mathrm{Bi}_{0.75} \mathrm{Y}_{0.25} \mathrm{FeO}_{3}$ calculated in this work and (shadow) $\mathrm{Bi}_{0.8} \mathrm{Y}_{0.2} \mathrm{FeO}_{3}$ obtained in Ref. 9 .

Table 1. Optimized structural parameters of $\mathrm{BiFeO}_{3}$ ground state ( $R 3 c \mathrm{G}$-AFM). The lattice constant of the rhombohedral primitive cell, $a$, is given in $\AA$, the rhombohedral angle, $\alpha$, in deg and the equilibrium unit cell volume, $V_{0}$, in $\AA^{3}$. The Wyckoff positions $2 a$ and $6 b$ are referenced to the rhombohedral system.

\begin{tabular}{cccr}
\hline Parameters & Present & Exp. Ref. [23] & Theo. Ref. [2] \\
\hline$a$ & 5.702 & 5.63 & 5.697 \\
$\alpha$ & 59.128 & 59.35 & 59.235 \\
$V_{0}$ & 128.51 & 124.6 & 128.48 \\
$\mathrm{Bi}(2 a)$ & $0 ., 0 ., 0$. & $0 ., 0 ., 0$. & $0 ., 0 ., 0$. \\
$\mathrm{Fe}(2 a)$ & $0.222,0.222,0.222$ & $0.221,0.221,0.221$ & $0.2232,0.2232,0.2232$ \\
$\mathrm{O}(6 b)$ & $0.536,0.937,0.391$ & $0.538,0.933,0.395$ & $0.5342,0.9357,0.3865$ \\
\hline
\end{tabular}

The localization of the morphotropic phase boundary (MPB) is highly desirable as at this boundary two crystallographic different phases, separated by low energy barriers, coexist. In general, the coexistence of two rather different phases in a multi-domain sample results in an enhancement of the electromechanical response, as it was predicted in the vicinity of a strain induced $R 3 c-P n m a$ morphotropic phase boundary in pristine $\mathrm{BiFeO}_{3}$ [30; 31]. The dissimilar physical properties of the two phases gives rise to giant responses when the phase transformation is in- duced, even with a slight external perturbation. To determine the Y-concentration $x$ at which the phase transition occurs, we take into account the energetics of the mentioned structural phases as a function of $x$. The different dopant configurations within each concentration introduce an uncertainty in the determination of the transition $x$-value. From the data plotted in figure 4 , the interval at which both the $R 3 c$ and the Pnma phases coexist can be calculated, giving $x=0.18 \pm 0.02$. From table 2 and figure 5 it can be seen that the bulk modulus as well as the unit cell 
Table 2. Ground state symmetry, equilibrium volume $\mathrm{V}_{0}$ (in $\AA^{3}$ ) and bulk modulus $\mathrm{B}_{0}$ (in GPa) for the studied Y-concentrations.

\begin{tabular}{cccc}
\hline Y-doping & Phase & $\mathrm{V}_{0}$ & $\mathrm{~B}_{0}$ \\
\hline 0.000 & $R 3 c$ & 64.53 & 99.76 \\
0.125 & $R 3 c$ & 63.87 & 106.95 \\
0.250 & Pnma & 61.00 & 163.22 \\
\hline
\end{tabular}

volume change abruptly when going from the $R 3 c$ to the Pnma phases at $x=0.18 \pm 0.02$, indicating a first order transition. As stated in Ref. 32, due to the Gibbs phase rule, there should exist an intermediate structural state within a concentration range close to the boundary where both phases coexist. It is within this concentration range where a tuning of the physical properties could be done.

The same phase transition is obtained when doping $\mathrm{BiFeO}_{3}$ with $30 \%$ of $\mathrm{La}[24 ; 33$; 34], which has a ionic radius similar to that of $\mathrm{Bi}^{3+}(1.16 \AA$ and $1.17 \AA$, respectively). In this case, the absence of the lone-pair in $\mathrm{La}^{3+}$ yields the preference towards a more isotropic and highly-coordinated A-cation, as it is the Pnma structure [35]. The same effect of lone-pair suppression as in the case of La doping is obviously introduced when doping with $\mathrm{Y}^{3+}$. On the other hand, as the ionic radius of $\mathrm{Y}^{3+}$ is smaller than the $\mathrm{Bi}^{3+}$ one, chemical pressure is induced in this case. The larger the Y-concentration, the bigger the induced chemical pressure and, therefore, it is reasonable to find analogous consequences and structural evolution when doping as when applying hydrostatic pressure to pristine $\mathrm{BiFeO}_{3}$ (See figure 2(a)). Therefore, when doping with yttrium the two mentioned effects sum up, namely chemical pressure and lone-pair absence, giving rise to the same phase transition reported for $\mathrm{Bi}_{1-x} \mathrm{La}_{x} \mathrm{FeO}_{3}$, but at a lower dopant concentration. To ilustrate the presence of both effects we show in Fig. 6 the local distortion around one yttrium comparing it with the pristine $\mathrm{BiFeO}_{3}$. It is already established that in the pristine $\mathrm{BiFeO}_{3}\left(\mathrm{ABO}_{3}\right)$, there are two different bonding lengths between the $\mathrm{A}(\mathrm{A}=\mathrm{Bi})$ atoms and the $\mathrm{O}$ atoms lying within the (111) planes, indicating the occurrence of permanent polarization in terms of a structural point of view. When introducing the Y-dopants, there are induced structural local distortions that modify these bonding lengths such that the larger A-O lengths become shorter and the shorter ones become larger, when $\mathrm{A}=\mathrm{Y}$ (See Fig. 6). This is a consequence of the lack of the lone-pair in yttrium. Besides, within our considered unit cell, we find the same behavior in the Bi atoms sorrounding the dopant, in average, thus reducing the off-center shift of the Bi atoms, which gives rise to a lower polarization value. Also from Fig. 6 it can be seen that the smaller ionic radious of yttrium induces a slight decrease in the Bi-Fe bonding-lengths, yielding a shrinking of the unit cell.

The electron localization function (ELF) gives an insight into the chemical bonding present in solids [36]. Low ELF values indicate a dominant ionic bonding character, while large values indicate the presence of a covalent bonding character. In order to analyze the incidence of Y-doping in the local bonding environment we show in Fig. 7 the ELF for two cases of $\mathrm{Bi}_{1-x} \mathrm{Y}_{x} \mathrm{FeO}_{3}(\mathrm{x}=0,0.25)$ within the $R 3 c$ structure. In the pristine case the lobe-like structure at the $\mathrm{Bi}$ sites indicates the presence of the 6 s lone-pair which is obviously absent at the $Y$ sites in the doped case. Besides, in the doped compound, the calculated ELF values show that the lobe-like structure becomes more symmetric at the Bi-sites, consistent with the previously mentioned lowering of the polarization value.

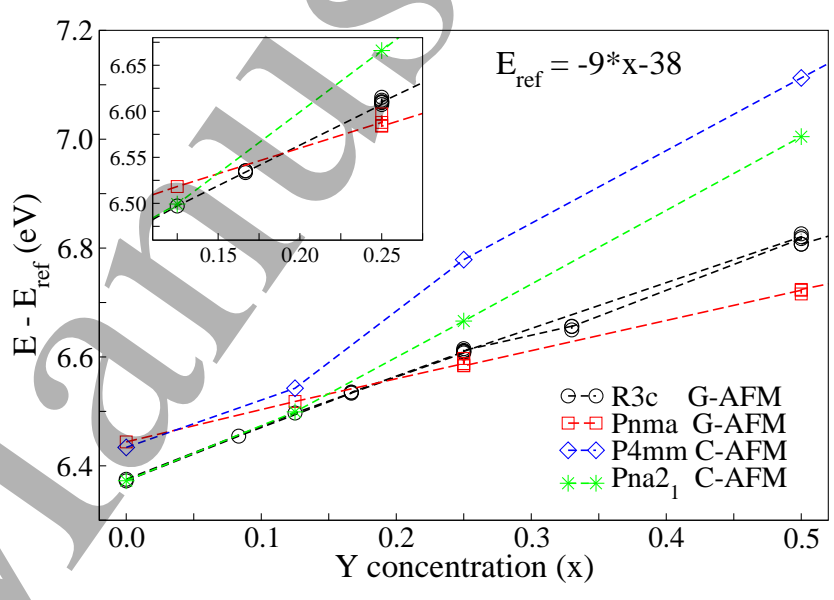

Figure 4. Energy of $\mathrm{Bi}_{1-x} \mathrm{Y}_{x} \mathrm{FeO}_{3}$ within the $R 3 c$, Pnma, Pna $2_{1}$ and P4mm phases, plotted as a function of the Y-concentration $x$ for different dopant arrangements, when it corresponds.To facilitate the visualization of the crossover, following Ref. 24 we define an energy reference $E_{r e f}(x)=-9 x-38$. The dashed-lines are drawn to guide the eye.

For completeness find in table 3 the energies of the lowest lying solutions for the considered cases, referenced to the corresponding ground state for each doping.

On hand of our results we also analyze the increased ferromagnetic response obtained by SalehMedina et al. upon doping $\mathrm{BiFeO}_{3}$ with yttrium. It is by now well stablished that the wFM shown by $\mathrm{BiFeO}_{3}$ has its origin in the Dzyaloshinsky-Moriya interaction (DMI), which competes with the exchange interaction. Exchange favors collinear magnetic structures, while the DMI favors perpendicular magnetic configurations between adjacent spins. According to Weingart et al. [37], the antiferrodistortive (AFD) distortions in $\mathrm{BiFeO}_{3}$ within the $R 3 c$ structure reduce the strength of the exchange interaction and 


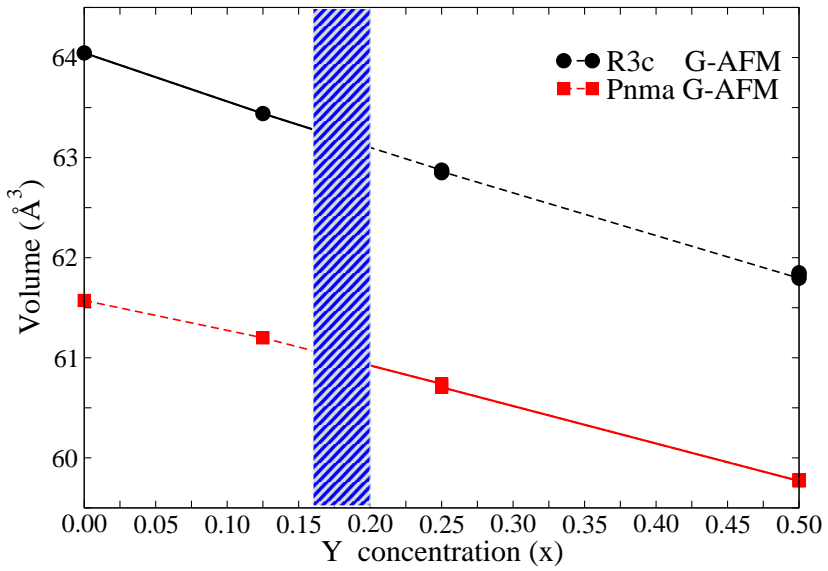

Figure 5. Volume per formula unit as a function of $\mathrm{Y}$-concentration (x) for the R3c and Pnma phases. The shadowed vertical stripe indicates the concentration range

at which the phase transition occurs.

Table 3. Energies (in meV) of the optimized considered phases for the different magnetic configurations. For each doping we fix the zero energy at the corresponding ground state.

\begin{tabular}{cccccc}
\hline System & Phase & G-AFM & C-AFM & A-AFM & FM \\
\hline \multirow{2}{*}{$\mathrm{BiFeO}_{3}$} & R3c & 0 & 59 & 129 & 211 \\
& Pnma & 69 & 125 & 194 & 271 \\
& Pna $2_{1}$ & 7 & 2 & 141 & 160 \\
& P4mm & 66 & 59 & 190 & 209 \\
\hline \multirow{3}{*}{$\mathrm{Bi}_{0.875} \mathrm{Y}_{0.125} \mathrm{FeO}_{3}$} & R3c & 0 & 58 & 127 & 208 \\
& $P n m a$ & 15 & 72 & 142 & 217 \\
& $P n a 2_{1}$ & 12 & 10 & 147 & 164 \\
& P4mm & 49 & 46 & 182 & 198 \\
\hline \multirow{3}{*}{$\mathrm{Bi}_{0.75} \mathrm{Y}_{0.25} \mathrm{FeO}_{3}$} & Rnc & 24 & 84 & 152 & 232 \\
& Pnma & 0 & 59 & 128 & 203 \\
& Pna $2_{1}$ & 29 & 24 & 221 & 235 \\
& P4mm & 202 & 195 & 348 & 366 \\
\hline \multirow{3}{*}{$\mathrm{Bi}_{0.5} \mathrm{Y}_{0.5} \mathrm{FeO}_{3}$} & R3c & 91 & 158 & 227 & 304 \\
& Pnma & 0 & 57 & 130 & 203 \\
& Pna $2_{1}$ & 291 & 289 & 441 & 457 \\
& P4mm & 403 & 397 & 566 & 583 \\
\hline & & & & &
\end{tabular}

enhance the DMI one. Furthermore, the single ion anisotropy (SIA), which couples the spin space with the spatial coordinates establishing a preferential direction (or plane) for the magnetic moments, should be also taken into account. The incidence of the SIA is not straightforward, because the FE distortions favor an easy axis while AFD distortions favor an easy plane (see Ref. 37). The small value of the magnetic anisotropy constant, leads to an easy plane perpendicular to the hard axis (111) in $\mathrm{BiFeO}_{3}-R 3 c$ [37]. When doping $\mathrm{BiFeO}_{3}$ with yttrium we find that, besides the lattice compression, the octahedral tilt gets larger with increasing Y-content, being of $11.5^{\circ}$ for $x=0$ and averaging to $(12.3 \pm 0.2)^{\circ}$ and $(13.0 \pm 0.3)^{\circ}$ for $x=0.125$ and $x=0.25$ respectively, within the $R 3 c$ structures.

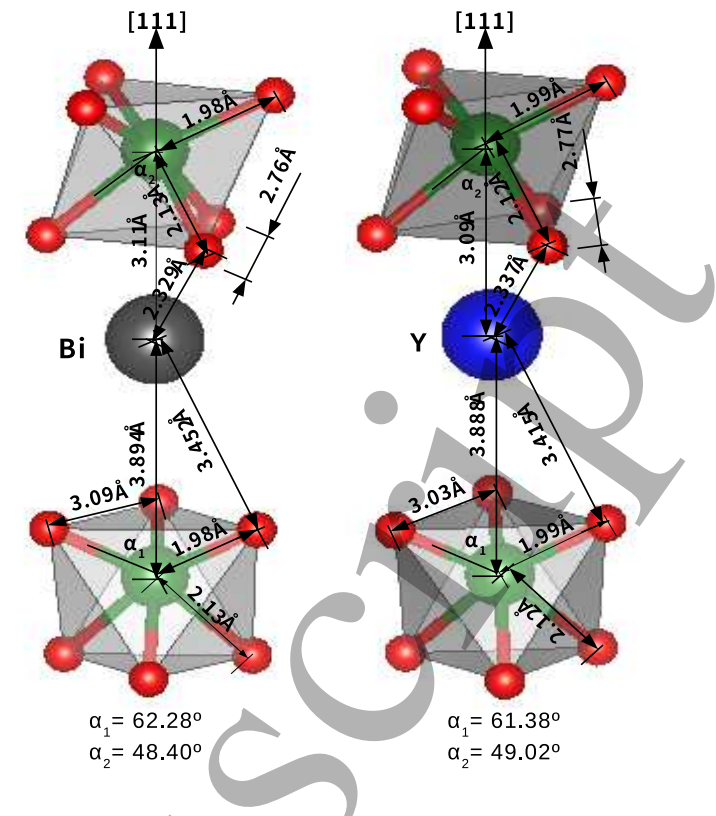

Figure 6. Local atomic structure centered at the $\mathrm{Bi}(\mathrm{Y})$-site of $\mathrm{BiFeO}_{3}$ $\left(\mathrm{Bi}_{1-x} \mathrm{Y}_{x} \mathrm{FeO}_{3}\right)$ lattice, showing the off-centering shift of the Bi-ion (Y-ion) along the [111] direction of the 40-atom unit cell.

Therefore, the AFD distortions are enhanced with increasing Y-concentration. We also find that the FE distortion progressively diminishes upon doping as the calculated polarizations are $P=94 \mu \mathrm{C} / \mathrm{cm}^{2}$ for $x=0$ and $P=89 \mu \mathrm{C} / \mathrm{cm}^{2}$ for $x=0.125$. Accordingly, we expect that the SIA should stabilize the easy plane perpendicular to the (111) direction in a cooperative way with the DMI. This cooperative distortions should enhance the ferromagnetic response, as it is experimentally observed in Ref. 9 for $\mathrm{Bi}_{1-x} \mathrm{Y}_{x} \mathrm{FeO}_{3}$ with $0 \leq x \leq 0.20$.

For $x=0.20$ a phase transition to the Pnma structure is experimentally found (supported by the results of this work) and also a significant change in the magnetic behavior at room temperature, i.e. a superparamagnetic behavior appears. This superparamagnetic response is beyond what these bulk calculations can explain. Actually, in Ref. 9 the particle size decreases with Y-doping and superparamagnetism seems to be a consequence of the particles volume decrease.

\section{Conclusions}

Recent experimental results on Y-doped $\mathrm{BiFeO}_{3}$ nanoparticles detected a structural phase transition for an yttrium concentration between $10 \%$ and $20 \%$. In this work we analyze, by means of DFT calculations, the possible transitions as a function of volume for pristine and doped bulk $\mathrm{BiFeO}_{3}$ in order to clarify and understand the experimental results. We find that, due to the smaller cation $\mathrm{Y}^{3+}$ size 


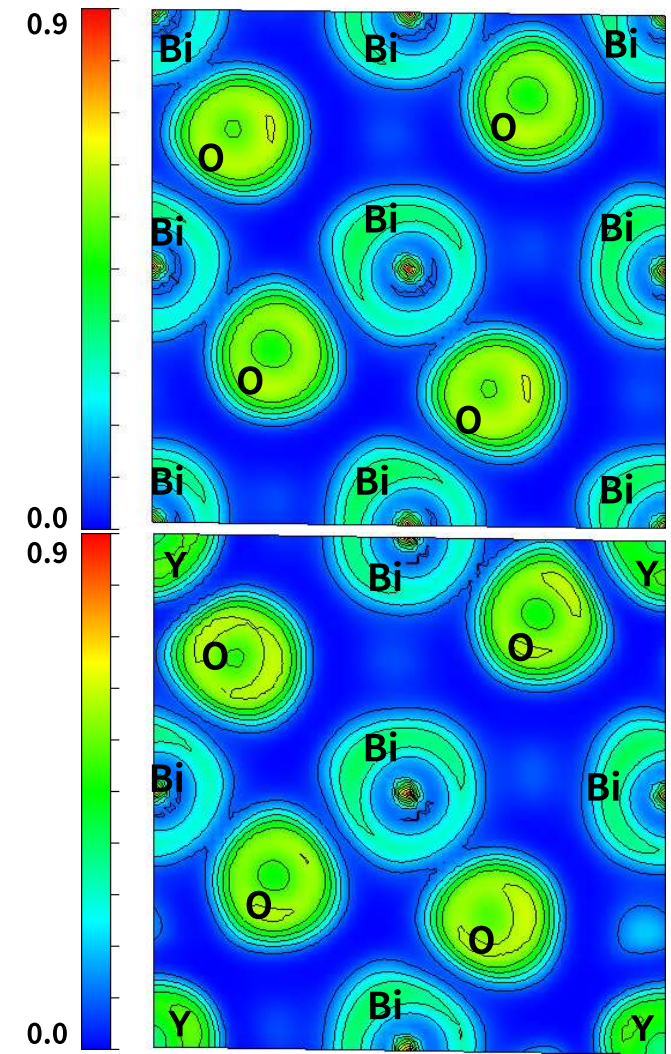

Figure 7. Electron localization function (ELF) plotted along the (100) plane for (left) pristine $\mathrm{BiFeO}_{3}$ and (right) $\mathrm{Bi}_{0.75} \mathrm{Y}_{0.25} \mathrm{FeO}_{3}$ within the $R 3 c$ structure.

and the partial suppression of the 6 s lone-pair, the local Fe environment is distorted with different $\mathrm{Fe}-\mathrm{O}$ bonds and $\mathrm{Fe}-\mathrm{O}-\mathrm{Fe}$ angles in such a way that at an $\mathrm{Y}$ concentration of $x=0.18 \pm 0.02$ a rombohedral $(R 3 c)$ to orthorhombic (Pnma) first order'structural phase transition should take place in bulk. This result is compatible with the experimental diffraction patterns reported in Ref. 9. The evolution of the octahedral tilts of the surrounding oxygen atoms with increasing Ydoping together with a smaller ferroelectric distorsion should lead to a larger canting of the Fe magnetic moments and to a larger ferromagnetic response in the $R 3 c$ phase, effect that is being also observed in the experiments. The superparamagnetic response experimentally detected beyond the structural phase transition could be attributed, on the other hand to low dimension effects of the Y-doped $\mathrm{BiFeO}_{3}$ nanoparticles.

\section{Acknowledgments}

This work was partially supported by PIP No 112201201-00069 of CONICET, and PICT 2014-1555 and
PICT 2015-0869 of the ANPCyT, Argentina.

\section{References}

[1] Teague J, Gerson R and James W 1970 Solid state Commun. 81073

[2] Ravindran P, Vidya R, Kjekshus A, ag H F and Eriksson O 2006 Phys. Rev. B 74224412

[3] Diéguez O, González-Vázquez O E, Wojdeł J C and Íñiguez J 2011 Phys. Rev. B 83094105

[4] Kiselev S, Ozerov R and Zhdanov G1963 Soviet Phys. Doklady 7742

[5] Wang J, Neaton J B, Zheng H, Nagarajan V, Ogale S B, Liu B, Viehland D, Vaithyanathan V, Schlom D G, Waghmare U V, Spaldin N A, Rabe K M, Wuttig M and Ramesh R 2003 Science 299 17191722 ISSN 0036-8075

[6] Hong N H, Sakai J, Poirot N and Brizé V 2006 Phys. Rev. B 73(13) 132404

[7] Hong NH, Thu Huong N, Kim T Y, Goumri-Said S and Kanoun M B 2015 The Journal of Physical Chemistry C 11914351-14357

[8] Xu Q, Zai H, Wu D, Qiu T and Xu M X 2009 Appl. Phys. Lett. 95112510

[9] Saleh-Medina L, Jorge G and Negri R 2014 J. Alloys Compd. 592306

[10] Zhong M, Kumar N P, Sagar E, Jian Z, Yemin H and Reddy P V 2016 Materials Chemistry and Physics 173126

[11] Wei J, Liu Y, Bai X, Li C, Liu Y, Xu Z, Gemeiner P, Haumont R, Infante I C and Dkhil B 2016 Ceramics International 4213395

[12] Fki H, Koubaa M, Sicard L, CheikhrouhouKoubaa W, Cheikhrouhou A and Ammar-Merah S 2017 Ceramics International 434139

[13] Mishra R, Pradhan D K, Choudhary R and Banerjee A 2008 J. Phys.: Condens. Matter 2045218

[14] Sheng Y, Rui W, Du J, Zhou S and Xu Q 2014 J. Appl. Phys. 115 17D902

[15] Luo L, Wei W, Yuan X, Shen K, Xu M and Xu Q 2012 J. Alloys Compd. 54036

[16] Wu Y K, Chen X K, Zhang J and Chen X J 2012 J.of Magn. and Mag. Mat. 3241348

[17] Blöchl P 1994 Phys. Rev. B 5017953

[18] Kresse G and Furthmüller J 1996 Phys. Rev. B 54 11169

[19] Kresse G and Joubert D 1999 Phys. Rev. B 591758

[20] Perdew J, Burke S and M M E 1996 Phys. Rev. Lett. 773865

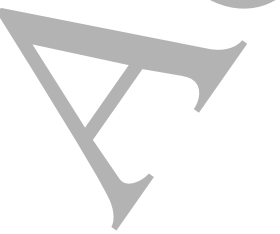


[21] Buhot J, Toulouse C, Gallais Y, Sacuto A, de Sousa R, Wang D, Bellaiche L, Bibes M, Barthélémy A, Forget A, Colson D, Cazayous M and Measson M A 2015 Phys. Rev. Lett. 115(26) 267204

[22] Michel C, Moreau J M, Achenbach G, Gerson R and James W 1969 Solid State Commun. 7701

[23] Kubel F and Schmid H 1990 Acta Crystallogr., Sect. B: Struct. Sci. 46698

[24] Lee J H, Oak M A, Choi H J, Son J Y and Jang H M 2012 J. Mater. Chem. 22 1667-1672

[25] Zeches R J, Rossell M D, Zhang J X, Hatt A J, He Q, Yang C H, Kumar A, Wang C H, Melville A, Adamo C, Sheng G, Chu Y H, Ihlefeld J F, Erni R, Ederer C, Gopalan V, Chen L Q, Schlom D G, Spaldin N A, Martin L W and Ramesh R 2009 Science 326977

[26] Kreisel J, Jadhav P, Chaix-Pluchery O, Varela M, Dix N, Sánchez F and Fontcuberta J 2011 J.Phys.:Condens. Matter 23342202

[27] Zhanga $Y X$, Wanga $X$, Wangc $Y$ and Wanga $Z$ 2010 J. Alloys Compd. 507157

[28] Levin I, Karimi S, Provenzano V, Dennis C L, Wu H, Comyn T P, Stevenson T J, Smith R I and Reaney I M 2010 Phys. Rev. B 81(2) 020103

[29] Kan D, Pálová L, Anbusathaiah V, Cheng C J, Fujino S, Nagarajan V, Rabe K and Takeuchi I 2010 Adv. Functional Materials 201108

[30] Sando D, Xu B, Bellaiche L and Nagarajan V 2016 Applied Physics Reviews 3011106

[31] Wang D, Salje E K H, Mi S B, Jia C L and Bellaiche L 2013 Phys. Rev. B 88(13) 134107

[32] Koval V, Skorvanek I, Durisin J, Viola G, Kovalcikova A, Svec P, Saksl K and Yan H 2017 J. Mater. Chem. C 52669

[33] S-TZhang, L-HPang, Zhang Y, M-HLu and Chen Y F 2006 J. Appl. Phys. 100114108

[34] S-TZhang, Zhang Y, M-HLu, C-LDu, Chen Y F, Liu Z G, Zhu Y Y, Ming N B and Pan X 2006 Appl. Phys. Lett. 88162901

[35] González-Vázquez O, Wojdel J C, Diéguez O and niguez J I 2012 Phys. Rev. B 85064119

[36] Savin A, Nesper R, Wengert S and Fssler T F 1997 Angew. Chem., Int. Ed. Engl. 361808

[37] Weingart C, Spaldin N and Bousquet E 2012 Phys. Rev. B 86094413

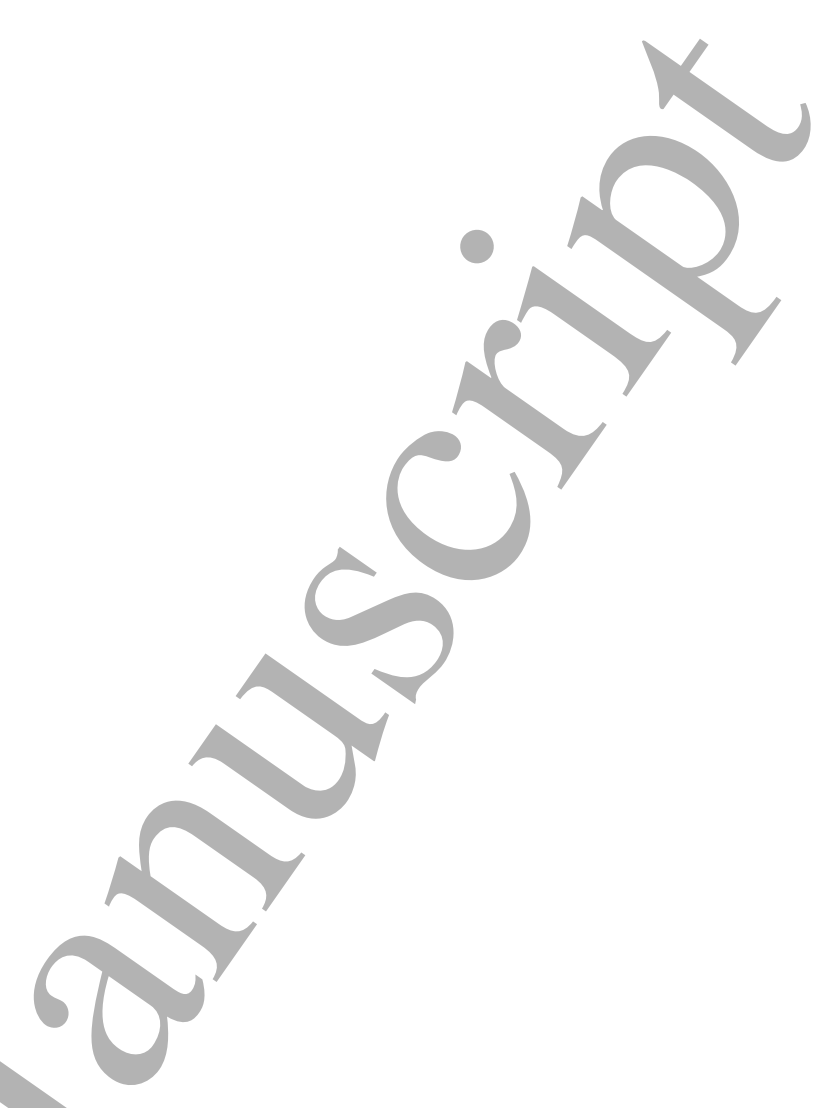

